\title{
Predischarge coronary angiography was better than exercise testing for reducing hospital use after low-risk chest pain
}

\author{
deFilippi CR, Rosanio $S$, Tocchi M, et al. Randomized comparison of a strategy of predischarge coronary angiography \\ versus exercise testing in low-risk patients in a chest pain unit: in-hospital and long-term outcomes. J Am Coll Cardiol \\ 2001 Jun 15;37:2042-9. \\ QUESTION: In low-risk patients evaluated for chest pain, does a strategy of predischarge \\ coronary angiography (CA) reduce repeated visits and admissions to the emergency \\ department (ED) more than a strategy of predischarge exercise treadmill testing (ETT)?
}

\section{Design}

Randomised \{allocation concealed $*\} \uparrow$, \{unblinded $\}$, * controlled trial with a median of 375 days of follow up.

\section{Setting}

A chest pain unit at the University of Texas Medical Branch at Galveston, Texas, USA.

\section{Patients}

248 patients 20 to 65 years of age with a $\leqslant 7 \%$ probability of acute myocardial infarction (MI) according to the Goldman algorithm, an absence of electrocardiographic and biochemical markers for ischaemia or infarction, an ability to exercise, and no previous coronary artery disease (CAD). Exclusion criteria were a previous CA or ETT or electrocardiogram confounders for ETT interpretation. $96 \%$ of patients completed follow up.

\section{Intervention}

123 patients were allocated to CA done with a standard femoral approach. CAD was defined as moderate if there was $50 \%$ to $69 \%$ lumen narrowing of a major epicardial artery or its branches and severe if there was $\geqslant 70 \%$ diameter stenosis. 125 patients were allocated to ETT done by using a symptom limited, standard, or modified Bruce protocol.

\section{Main outcome measures}

Main outcomes were return to the $\mathrm{ED}$ with a chief symptom of chest pain, admission for chest pain, death, and MI.
Source of funding: in part, Roche

Diagnostics.

For correspondence: $\mathrm{Dr}$

CR deFilippi,

University of

Maryland, Department

of Medicine, Division of

Cardiology, Room

G3K63, 22 South

Greene Street,

Baltimore, $M D$

21201-1595, USA

cdefilip@

medicine.umaryland.edu.

\section{Main results}

Analysis was by intention to treat. $19 \%$ of patients in the CA group had CAD, and 7\% of patients in the ETT group had a positive ETT result. Of the patients with negative or non-diagnostic test results who completed follow up $(\mathrm{n}=208)$, fewer of those who received CA rather than ETT returned to the $\mathrm{ED}$ with chest pain $(\mathrm{p}<0.001)$ or were admitted to the hospital at 1 year $(\mathrm{p}=0.003)$ (table). No deaths or MIs occurred in either group. When all patients were considered (ie, those with positive or negative test results), a greater cumulative proportion of patients in the CA group than in the ETT group remained free of ED returns at 1 year $(83 \% v 69 \%, \mathrm{p}=0.041)$.

Predischarge coronary angiography (CA) v exercise treadmill testing (ETT) for low-risk chest pain

\begin{tabular}{lrrlll} 
Outcomes at 1 year & CA & ETT & RRR (95\% CI) & NNT (CI) \\
\hline Return ED visit for chest pain & $10 \%$ & $30 \%$ & $66 \%(36$ to 82$)$ & 6 (4 to 11$)$ \\
\hline Admission for chest pain & $3 \%$ & $16 \%$ & $81 \%(43$ to 94$)$ & $8(5$ to 18$)$ \\
\hline
\end{tabular}

¥Abbreviations defined in glossary; RRR, NNT, and $\mathrm{Cl}$ calculated from data in article.

\section{Conclusion}

Predischarge coronary angiography reduced emergency department visits and hospital admissions more than did predischarge exercise treadmill testing in low-risk patients evaluated in the emergency department for chest pain.

*See glossary.

$\dagger$ Information provided by author.

\section{COMMENTARY}

ETT has been a mainstay of ED protocols for low-risk patients with chest pain in the USA for over a decade. ${ }^{1}$ The trial by deFilippi et al considered CA as an alternative acute testing strategy. Table 1 of the original article shows that a higher percentage of patients in the CA group (with positive or negative CA results) than in the ETT group (with positive or negative results) had chest pain features typical of ischaemia. This finding may partially account for the greater prevalence of disease detected by CA than by ETT.

Patients with negative ETT results were twice as likely as those with negative CA results to believe that recurrent chest pain was of cardiac origin $(15 \% v 7 \%)$, which may have influenced their readiness to return to an ED. More complete identification and effective management of patients with $\mathrm{CAD}$ may also have played a role in decreasing service use in the group with negative CA results. Fewer patients in this group reported frequent recurrent chest pain than did those in the group with negative ETT results (21\% v 35\%).

In an earlier observational study, ${ }^{2} 17 \%$ of patients with negative ETT results returned to the ED, and $12 \%$ were admitted to the hospital within 6 months of discharge from an ED according to a chest pain protocol. In that study, and in the trial by deFilippi $e t a l$, more patients with positive test results on ETT or CA had invasive procedures. It is unclear whether these additional procedures prevented cardiac events.

Cost effectiveness cannot be assessed in the absence of hard data on the effectiveness of either CA or ETT in averting mortality and morbidity in low-risk patients. Increased use of CA for such patients could be selective or delayed. The appropriateness of emergency diagnostic strategies involving ETT or CA for this patient group is dependent on patients' and practitioners' values and expectations, and on the availability of medical technology in specific practice settings.

Peter C Wyer, MD Columbia University New York, New York, USA

1 Stein RA, Chaitman BR, Balady GJ, et al. Safety and utility of exercise testing in emergency room chest pain centers: an advisory from the Committee on Exercise, Rehabilitation, and Prevention, Council on Clinical Cardiology, American Heart Association. Circulation 2000;102:1463-7.

2 Polanczyk CA, Johnson PA, Hartley LH, et al. Clinical correlates and prognostic significance of early negative exercise tolerance test in patients with acute chest pain seen in the hospital emergency department. Am J Cardiol 1998;81:288-92. 